\title{
EFFICIENCY AND LIMITATIONS OF DECOMPRESSIVE CRANIECTOMY IN PATIENTS AFTER TRAUMATIC BRAIN INJURY - PRELIMINARY RESULTS
}

\author{
HANKo M, Richterova R, KolaROvSZKi B.
}

Clinic of Neurosurgery, Jessenius Faculty of Medicine in Martin, Comenius University in Bratislava, University Hospital Martin, Slovakia

\begin{abstract}
Introduction: Decompressive craniectomy (DC) has been recently proven effective tier II therapeutic procedure in the treatment of refractory posttraumatic intracranial hypertension. However, its full potential and effectivity is yet to be described and this surgery remains controversial. The goals of our study include analysis of efficiency of $\mathrm{DC}$ and description of risk factors associated with unfavourable outcome.

Methods: 24 patients who underwent DC at the Clinic of Neurosurgery, JFM CU in Martin, during years 2015-2016 were prospectively observed. Selected demographic, clinical, and radiographic factors were analysed and compared with patient's GOS (Glasgow Outcome Scale) at the time of their first ambulatory control (after 3.5 months in average).

Results: We observed mortality of $29.17 \%$. Good outcome (GOS 4-5) was achieved by $29.17 \%$ of the patients as well. Preoperative GCS $\leq 5(p=0.049)$, intraventricular bleeding $(p=0.0268)$, midline shift $\geq 15 \mathrm{~mm}(\mathrm{p}=0.0067)$, and the volume of intracranial lesion $(R=-0.41, p=0.046)$, especially its extracerebral component $(R=-0.46, p=0.02)$, were identified as statistically significant negative prognostic factors.

Conclusion: DC is effective in the management of patients with traumatic brain injury. Good outcome is achieved by $29.17 \%$ of the patients. Described negative prognostic factors (preoperative GCS $\leq 5$, intraventricular bleeding, midline shift $\geq 15 \mathrm{~mm}$, and increasing the volume of traumatic mass lesion) could help in targeting this surgery only to patients who are expected to benefit from it.
\end{abstract}

Keywords: intracranial hypertension, craniectomy, traumatic brain injury, outcome

\section{INTRODUCTION}

Decompressive craniectomy (DC) is a neurosurgical procedure designed to decrease and control excessively elevated intracranial pressure (ICP) (1). It is used in the treatment of intracranial hypertension following traumatic brain injury, malignant middle cerebral artery infarction, subarachnoid haemorrhage, inflammatory brain diseases, and, occasionally, for various other causes of elevated ICP (14). During this surgery cranial bone flap is removed (Fig. 1) and durotomy is performed so that formerly closed intracranial space with limited volume is opened and the brain is allowed to expand through the bony opening, thus lowering the ICP (1). Later, after the brain oedema is regressed and patient's condition is stabilized (usually following months), the bone flap is replaced (14).

It has been proven that DC reduces the elevated ICP (3) and improves cerebral blood flow (22) and tissue oxygenation (20). Despite these findings the role of DC in the treatment of traumatic brain injury is still considered controversial because of lack of complete Level I evidence and various results published in smaller, mostly retrospective, studies. Indicating criteria for DC are also not clearly defined. The surgery is currently indicated either as primary (early, prophylactic, or following evacuation of traumatic mass lesion) or secondary (in refractory intracranial hypertension) (14).

Address for correspondence:

Branislav Kolarovszki, Assoc. Prof. MD. PhD. Clinic of Neurosurgery, Jessenius Faculty of Medicine and University Hospital, Martin, Kollarova Str. N. 2, 036 59, Martin, Slovakia

e-mail: kolarovszki@jfmed.uniba.sk 




Fig. 1 Recommended extent of bifrontal DC (left) and hemicraniectomy (right)

Prospective multicentric randomised study RESCUEicp reported mortality of $29.9 \%$ and favourable outcome of $42.8 \%$ following 6 months after secondary DC for refractory posttraumatic intracranial hypertension (8). Primary DC following evacuation of acute subdural hematoma is being a subject of research in randomised multicentric prospective study RESCUE-ASDH (12). The Brain Trauma Foundation does not recommend the use of bifrontal DC in the treatment of posttraumatic diffuse brain injury and refractory ICP $>20$ $\mathrm{mmHg}$ for more than 15 minutes and recommends a large (at least $12 \times 15 \mathrm{~cm}$ ) decompressive hemicraniectomy over a smaller one (2). Until a complete Level I evidence for efficacy of DC is obtained and proper indicating criteria are established there is going to be a substantial need for further research in this field.

\section{METHODS}

We prospectively observed 24 patients who underwent DC for traumatic brain injury at the Clinic of Neurosurgery JFM CU during years 2015-2016. In all cases the surgery consisted of unilateral fronto-temporo-parietal craniectomy with augmentative duraplasty. Patients with infratentorial traumatic lesion, patients younger than 18 years, and patients who later suffered additional injury on the site of DC were excluded from this study. Possible demographic, clinical, and radiographic predictive factors were assessed.

Demographic factors included patients' age and sex, clinical factors consisted of preoperative GCS, absence of pupillary reflex (without regards to its side), and consumption of alcohol before the injury. Radiographic analysis was based on CT findings and assessed the type of intracranial lesion, its volume, presence of intraventricular and/or subarachnoid bleeding (SAH), skull fracture, presence of midline shift (shift $\leq 5 \mathrm{~mm} ; 6-14 \mathrm{~mm}$ and $\geq 15 \mathrm{~mm}$ was differentiated), and compression of perimesencephalic cisterns (we distinguished intact, partially compressed, or completely obliterated cisterns).

These findings were later compared with patients' outcome quantified by means of Glasgow Outcome Scale (GOS), which is an international standardized measure with values 
ranging from 1 to 5 (21). Average (avg.) time of follow-up was 3.5 months, what represented the time of patients' first ambulatory control. Statistical analysis was performed using nonparametric tests: Mann-Whitney and Spearman correlation test.

\section{RESULTS}

The observed group of 24 patients consisted of 17 men (70.83\%) and 7 women (29.17\%). The avg. age was 58.8 years. In time of first ambulatory control (3.5 months after surgery on avg.) the patients' outcome was following (Graph 1):

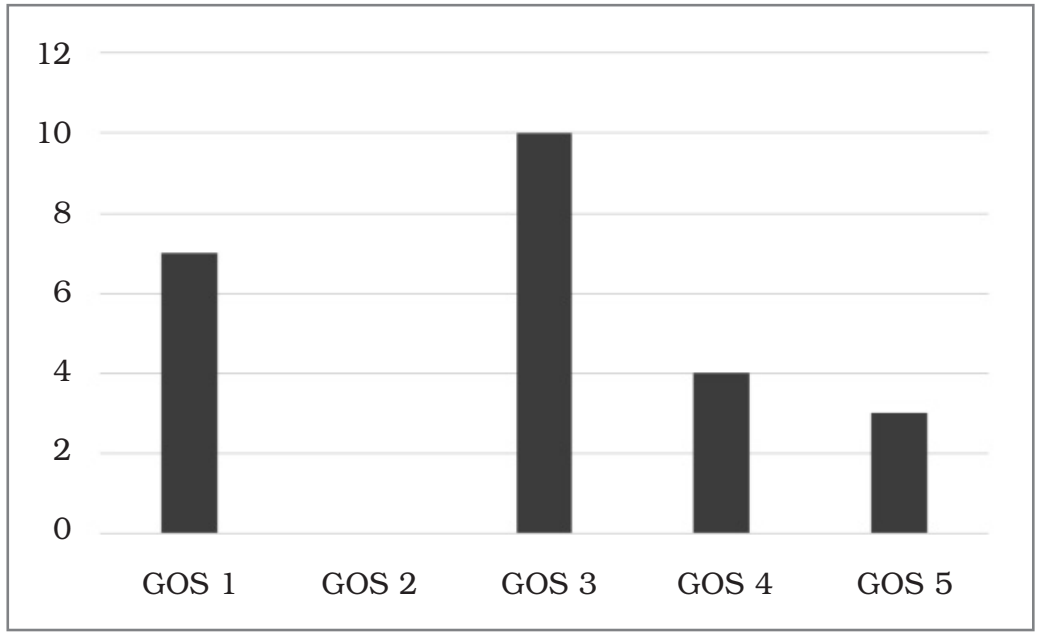

Graph 1 Outcome of our patients $(n=24)$ after DC

Favourable outcome (GOS 5-4 representing no or mild disability) was achieved by 7 (29.17\%) patients with 3 achieving GOS 5 and 4 achieving GOS 4. Severe disability (GOS 3) persisted in $10(41.67 \%)$ patients and permanent vegetative state (GOS 2) was not observed in our cohort. Mortality represented by GOS 1 was $29.17 \%$ (7 patients).

Analysis of risk factors resulted in these findings: We did not observe significant correlation between patients' age and the outcome $(\mathrm{R}=-0.109 ; \mathrm{p}=0.61$ in Spearman test); however, patients who were 70 and more years old had the highest mortality of $60.0 \%$ and avg. GOS of $2 \pm 1.26$. (Tab. 1)

Tab. 1 Relationship between outcome and patients`age $(n=24)$

\begin{tabular}{|l|c|c|c|c|c|}
\hline age (decade) & 3. & 4. & 5. & 6. & 7. \\
\hline number (\%) & $3(12.5)$ & $3(12.5)$ & $4(16.67)$ & $9(37.5)$ & $5(20.83)$ \\
\hline mortality (\%) & 33.3 & 33.3 & 0.0 & 22.2 & 60.0 \\
\hline avg. GOS & 3.0 & 2.57 & 3.25 & 3.11 & 2.0 \\
\hline SD & 2.0 & 1.52 & 0.5 & 1.37 & 1.26 \\
\hline
\end{tabular}


Patients' sex did not result in significant difference in outcome as men achieved avg. GOS $2.88 \pm 1.45$ and women achieved avg. GOS $2.71 \pm 1.25$ ( $\mathrm{p}=0.436$ in Mann-Whitney test). Patients with preoperative GCS $\leq 5$ achieved a significantly worse outcome than the rest (avg. GOS $1.83 \pm 1.33$ vs. $3.17 \pm 1.56$ respectively, $\mathrm{p}=0.049$ in Mann-Whitney test), however we did not confirm significant negative correlation between patients' GCS and outcome $(\mathrm{R}=0.29, \mathrm{p}=0.16$ in Spearman test). Prior to injury alcohol was consumed by $11(45.8 \%)$ patients and we did not observe significant difference in their outcome when compared with the remaining patients (avg. GOS $2.82 \pm 1.33$ vs. $2.85 \pm 1.46$ respectively, $\mathrm{p}=0.49$ in Mann-Whitney test). 8 patients presented with absence of pupillary reflex, what resulted in avg. GOS $2.5 \pm 1.3$, but this result was not statistically significant ( $p=0.316$ in Mann-Whitney test) when compared with the others (avg. GOS $3.0 \pm 1.41$ ).

Radiographic analysis resulted in 14 patients with combination of intra- and extracerebral traumatic mass lesion having the worst outcome (avg. GOS $2.36 \pm 1.3$ ), 2 patients with isolated intracerebral mass lesion having avg. GOS $3.5 \pm 0.7$, and patients with extracerebral bleeding (acute subdural hematoma - ASDH in all 8 cases) scoring avg. GOS 3.5 \pm 1.3 . Statistical analysis could not be done in this observation due to insufficient number of patients in group with intracerebral lesion. (Fig. 2)



Fig 2 Illustration of CT findings: A - extracerebral lesion (ASDH), B - intracerebral lesion (haemorrhagic contusion), $\mathrm{C}$ - combined lesion (ASDH + haemorrhagic contusion)

Midline shift $\geq 15 \mathrm{~mm}$ in 7 patients resulted in a significantly worse outcome when compared with the other 17 patients, avg. GOS $1.7 \pm 1.2$ and mortality of $71.43 \%$ vs. $2.83 \pm$ 1.37; $\mathrm{p}=0.0067$ in Mann-Whitney test. 2 patients with complete obliteration of perimesencephalic cisterns had GOS 1, partial compression in 14 patients resulted in avg. GOS $3.07 \pm 1.33$, and 8 patients with intact cisterns had avg. GOS $2.88 \pm 1.36$; however, these findings were not confirmed to be statistically significant. Thirteen patients with skull fracture had avg. GOS $2.54 \pm 1.39$ compared to avg. GOS 3.0 \pm 1.25 in remaining patients and this difference was not statistically significant $(\mathrm{p}=0.575$ in Mann-Whitney test). Presence of SAH resulted in avg. GOS $2.57 \pm 3.2$ in 14 patients vs. avg. GOS $1.34 \pm 1.4$ in remaining 10 patients, but this was not statistically significant ( $\mathrm{p}=0.749$ in Mann-Whitney test). Presence of intraventricular bleeding was confirmed to be a significant negative prognostic factor as 5 patients with hematocephalus had avg. GOS $1.4 \pm 0.89$ vs. avg. GOS $3.21 \pm 1.23$ in the group of other 19 patients ( $p=0.0268$ in Mann-Whitney).

Total volume of traumatic intracranial bleeding negatively correlated with patients' outcome $(\mathrm{R}=-0.41 ; \mathrm{p}=0.046$ in Spearman test) and similar findings were recorded during the analysis of extracerebral lesion's volume $(R=-0.46 ; p=0.02$ in Spearman test). On the other 
hand, the volume of intracerebral mass lesion did not correlate with patients' outcome $(\mathrm{R}=$ -0.23; $\mathrm{p}=0.275$ in Spearman test). (Tab. 2)

Tab. 2 Volumetric analysis of traumatic intracranial mass lesions $(n=24)$

\begin{tabular}{|l|l|c|c|c|c|c|}
\hline \multicolumn{2}{|c|}{ GOS } & 5 & 4 & 3 & 2 & 1 \\
\hline \multirow{4}{*}{$\begin{array}{l}\text { Average } \\
\text { volume } \\
\left(\mathbf{c m}^{3}\right)\end{array}$} & Extracerebral component & 40.43 & 52.08 & 48.88 & - & 106.04 \\
\cline { 2 - 7 } & SD & 34.01 & 51.21 & 48.92 & - & 56.94 \\
\cline { 2 - 7 } & Intracerebral component & 17.0 & 15.15 & 58.01 & - & 27.8 \\
\cline { 2 - 7 } & TD & 29.44 & 28.14 & 53.56 & - & 31.6 \\
\cline { 2 - 7 } & Total & 57.43 & 67.23 & 106.9 & - & 121.33 \\
\cline { 2 - 7 } & SD & 35.32 & 36.89 & 44.37 & - & 65.61 \\
\hline
\end{tabular}

\section{DISCUSSION}

The results we report (mortality $29.17 \%$ and the same number of patients with favourable outcome) are similar to those published in numerous other studies regarding the efficiency of DC. However various authors use various definitions for favourable outcome, which was defined as GOS 4-5 in our study. Guerra et al. observed mortality in $19 \%$ and good outcome in $58 \%$ of their patients and this was one of first reports regarding success of DC after traumatic brain injury [5], Juráň et al. reported favourable outcome in $26 \%$ of patients (11). Very similar observations were made in study RESCUEicp with mortality of $26.9 \%$ and good outcome of $27.4 \%$, but we must consider that these results were obtained only from patients after secondary DC (8).

Age was described as a negative prognostic factor $(6,16)$, but possibly due to a small number of patients we were unable to confirm this in our report. Still, patients who were 70 and more years old had the worst outcome. Patients' sex did not result in a significantly different outcome and this is in accordance with the results of a meta-analysis of Husson et al. from 2010 (7). Preoperative GCS is a well-known negative prognostic factor (7) and in our study GCS $\leq 5$ resulted in a significantly worse outcome. Consumption of alcohol prior to injury probably does probably not play a role in patients' outcome (18), recent meta-analysis presented by Raj et al. surprisingly showed a better outcome in these patients, but explanation of such result is uncertain (17). In our report alcohol did not influence the outcome of our patients but we did not consider its blood level. Absence of pupillary reflex has been repeatedly reported as a negative prognostic factor $(9,19)$. Possibly because of a limited number of patients with non-reactive pupils and the fact that uni- or bilaterality of this was not taken into account we did not observed similar result in our group of patients.

ASDH has been described as a negative prognostic marker (7). We were not able to obtain similar result possibly due to a small number of patients but ASDH was present in 21 of our patients and in 14 patients it occurred in combination with intracerebral traumatic mass lesion, what resulted in the worst outcome. Volumetric analysis of traumatic intracranial mass lesions of our patients showed that the total volume of intracranial traumatic bleeding, especially its extracerebral component, negatively correlates with patients' outcome. The volume of traumatic mass lesion is considered to be negative prognostic factor 
in patients with brain injury [9]. Midline shift is definitely a considerable negative prognostic mark $(7,19)$ and it is even one of the indicating criteria for evacuating of traumatic mass lesion (4). In our group midline shift $\geq 16 \mathrm{~mm}$ resulted in a significantly worse outcome. We observed GOS 1 in both patients with completely obliterated perimesencephalic cisterns but we were not able to statistically prove this finding as a negative predictor. Still, obliteration of these cisterns is reported to be a strong negative prognostic factor $(15,10)$ and different results in our study are again possibly due to a limited number of patients involved. Presence of skull fracture is not considered to predict negative outcome and despite of fact that it could give us limited information about the force of impact during patients' injury it did not result in a worse outcome in our group of patients as well. SAH was not proven to be a negative prognostic factor in our group. The extent of SAH as a negative prognostic factor is reported in scientific literature (13). Bleeding into ventricular system resulted in mortality of $80 \%$ and avg. GOS 1.4 and it was a significant risk factor in our study. Meta-analysis by Husson et al. from 2010 does not consider this to be a negative prognostic factor (7), however the study from 2013 reported an opposite finding (10).

Observation that a considerable number of patients who underwent DC for traumatic brain injury survives with severe disability urges us to indicate DC only to patients who should be able to benefit from it. Optimal indicating criteria for DC could be specified with the use of negative prognostic factors. Signs suggesting unfavourable outcome in our group are described above and further research including larger number of patients can be expected to provide a more accurate knowledge.

\section{CONCLUSION}

Decompressive craniectomy is an effective procedure in the management of patients with traumatic brain injury. In our study a good outcome (GOS 4-5) was achieved by 29.17\% of the patients and we observed mortality of $29.17 \%$ as well. Also there is a substantial number of patients who survive with severe disability (41.67 \% for GOS 3). Our findings suggest that factors predicting unfavourable outcome include:

- preoperative GCS $\leq 5$

- midline shift $\geq 15 \mathrm{~mm}$

- obliteration of perimesencephalic cisterns

- volume of intracranial bleeding, especially its extracerebral part.

Personalisation of indicating criteria for DC in patients after severe brain injury could define the patients who can have the real benefit from this surgery.

\section{REFERENCES}

1. Balan C, Alliez B. Decompressive craniectomy - from option to standard - part I. Romanian neurosurgery $2009 ; 16$ (2):20-26.

2. Carney $\mathrm{N}$ et al. Guidelines for the Management of Severe Traumatic Brain Injury, Fourth Edition. Neurosurgery 2017; 80(1):6-15.

3. Cooper DJ et al. Decompressive craniectomy in diffuse traumatic brain injury. N Engl J Med 2011; 364 (16):1493-1502.

4. Greenberg MS. Handbook of Neurosurgery. New York: Thieme; 2016.

5. Guerra WK et al. Surgical decompression for traumatic brain swelling: indications and results. J Neurosurg 1999; 90(2):187-196.

6. Hukkelhoven CWPM et al. Patient age and outcome following severe traumatic brain injury: an analysis of 5600 patients. J Neurosurg 2003; 99(4):666-673.

7. Husson EC et al. Prognosis of six-month functioning after moderate to severe traumatic brain injury: A systematic review of prospective cohort studies. J Rehabil Med 2010; 42(5): 425-436. 
8. Hutchinson PJA et al. Trial of Decompressive Craniectomy for Traumatic Intracranial Hypertension. N Engl J Med 2016; 375:1119-113.

9. Jacobs B et al. Computed Tomography and Outcome in Moderate and Severe Traumatic Brain Injury: Hematoma Volume and Midline Shift Revisited. J Neurotrauma 2011; 28(2):203-215.

10. Jacobs B et al. Outcome Prediction in Moderate and Severe Traumatic Brain Injury: A Focus on Computed Tomography Variables. Neurocrit Care 2013; 19(1):79-89.

11. Juráň V. et al. Indikace dekompresivní kraniektomie u traumat mozku. Cesk Slov Neurol N 2009; 72/105(5): 439-445.

12. Kolias AG et al. Decompressive craniectomy following traumatic brain injury: developing the evidence base. Br J Neurosurg 2016; 30(2):246-250.

13. Lin TK, Tsai HC, HHsieh TC. The impact of traumatic subarachnoid hemorrhage on outcome: a study with grouping of traumatic subarachnoid hemorrhage and transcranial Doppler sonography. J Trauma Acute Care Surg 2012;73:131-136.

14. Mraček J. Dekompresivní kraniektomie. Praha: Galén; 2016.

15. Perel PA et al. Predicting outcome after traumatic brain injury: practical prognostic models based on large cohort of international patients. BMJ 2008; 336:425.

16. Potts MB et al. Predictive values of age and the Glasgow Coma Scale in traumatic brain injury patients treated with decompressive craniectomy. Acta Neurochir Suppl (Wien) 2008; 102:109-112.

17. Raj R et al. Alcohol and mortality after moderate to severe traumatic brain injury: a meta-analysis of observational studies. J Neurosurg 2016; 124(6):1684-1692.

18. Rundhaug NP et al. Moderate and severe traumatic brain injury: effect of blood alcohol concentration on Glasgow Coma Scale score and relation to computed tomography findings. J Neurosurg 2015: 122(1):211-218.

19. Saade $\mathrm{N}$ et al. Evaluation of prognostic factors of decompressive craniectomy in the treatment of severe traumatic brain injury. Rec Col Bras Cir 2014; 41(4): 256-262.

20. Stiefel MF et al. Cerebral oxygenation following decompressive hemicraniectomy for the treatment of refractory intracranial hypertension. J Neurosurg 2004; 101 (2):241-247.

21. Teasdale GM. The Glasgow Coma and Outcome scales: Practical questions and answers. In: Sindou M, editor. Practical Handbook of Neurosurgery from Leading Neurosurgeons. Wien: Springer: 2009. p. 395-409.

22. Yamakami I, Yamaura A. Effects of decompressive craniectomy on regional cerebral blood flow in severe head trauma patients. Neurol Med Chir (Tokyo) 1993; 33(9):616-620.

\section{ABBREVIATIONS:}



Received: September, 21, 2017

Accepted: December, 1, 2017 